\title{
Hacia el futuro en cuidados intensivos pediátricos
}

\section{Towards the future in pediatric intensive care}

\section{E. Ocete Hita}

Servicio de Pediatría, Unidad de Cuidados Intensivos Pediátricos, Hospital Universitario Virgen de las Nieves, Granada, España

La medicina intensiva pediátrica puede, aún hoy en el año 2011, considerarse como una especialidad «nueva» y con un futuro prometedor. Desde el inicio del desarrollo de las técnicas específicas de asistencia y control de funciones vitales en niños y tipificación de salas diferenciadas para este fin hasta la estructuración de la disciplina como tal, no han transcurrido más de tres décadas. Sin embargo, en este período, se ha producido una evolución biotecnológica tal que los cuidados intensivos pediátricos han cambiado en gran medida sus conceptos, han adoptado nuevas metodologías y nos han impuesto, a los que nos dedicamos a esta especialidad, una puesta al día constante.

Buenas muestras de ello son: el incremento del número y, sobre todo, de la calidad de las unidades asistenciales dedicadas a los niños en situación crítica; la tipificación de técnicas y sistemas concebidos para niños; el incremento de bibliografía contrastada; la inclusión en los nuevos planes de estudios de temas dedicados a las urgencias vitales en pediatría y la calidad y cantidad de programas de formación específicos de cuidados intensivos pediátricos.

Existe una simbiosis evidente entra la medicina intensiva de adultos y la pediátrica. Las unidades de cuidados intensivos pediátricos (UCIP) han ido absorbiendo bagaje y experiencias de las $\mathrm{UCl}$ de adultos, modificando y adaptando protocolos, ajustando técnicas y diseñando materiales apropiados al múltiple abanico de edades, tamaños y pesos que abarca la edad infantil. Pero, en la actualidad, también se puede afirmar que, a través de «nuestros niños», en las $\mathrm{UCI}$ de adultos se han desarrollado nuevas y actuales técnicas ventilatorias, como la aplicación de la ventilación de alta frecuencia; técnicas de monitorización incruenta de gases, de medición de presiones (presión intracraneal

Correo electrónico: estherocete@ugr.es transfontanelar, que permitió el desarrollo de los sensores de fibra óptica); nuevas fórmulas farmacológicas, como el surfactante exógeno, etc.

En medicina intensiva pediátrica, del predominio de las enfermedades infecciosas y metabólicas (deshidrataciones), enfermedad aguda cardiológica, respiratoria, tratamientos postoperatorios de cirugía pediátrica general, cardiovascular, neurológica, etc., se ha pasado a unas enfermedades que exigen una atención más compleja, como los traumatismos craneoencefálicos severos, cirugía de trasplantes de órganos sólidos, afección secundaria a la inmunosupresión, etc. ${ }^{1}$. Muestra de ello son los artículos sobre traumatismo craneoencefálico que el grupo del Complejo Hospitalario Universitario Insular Materno-Infantil de Las Palmas de Gran Canaria publica en las páginas de este número de la revista MEDICINA INTENSIVA ${ }^{2,3}$.

En la actualidad, al desaparecer muchas de las enfermedades antes referidas y mejorar la asistencia primaria, los criterios de hospitalización pediátrica general y de ingreso en las UCIP han cambiado totalmente, estableciendo una nueva demanda asistencial al niño críticamente enfermo.

El trabajo asistencial con los niños susceptibles de cuidados intensivos conlleva varios aspectos: a) valoración clínica integral del niño, viéndole como un todo y no como el fallo de un órgano determinado; b) aplicación de una serie de técnicas y procedimientos diagnósticos y terapéuticos específicos para conocer el problema agudo, sus repercusiones y aplicarle los tratamientos oportunos, y c) continuidad en el seguimiento del proceso pues la evolución de la enfermedad varía constantemente y la adecuación de las normas terapéuticas constituye el trabajo sistemático en las UCIP. Todo ello condiciona en el personal médico la necesidad de tener unos conocimientos pediátricos profundos y dominar unas habilidades tecnológicas, capacidades de observación y toma de decisiones urgentes. 
En el futuro, se debe progresar en la «no invasividad», tanto en las técnicas de monitorización como en las terapéuticas, para reducir los complicaciones inherentes a las técnicas invasivas. En este sentido, el desarrollo actual de la monitorización de la función hemodinámica y cerebral es muy prometedor.

Para garantizar una buena rentabilidad en la asistencia pediátrica intensiva será cada vez más necesario, tener en cuenta aspectos clínicos (número de pacientes, tipo de enfermedad, técnica que se realiza, etc.) y parámetros administrativos que confirmen una buena relación costo/beneficio (índice de ocupación, estancia media, rotación paciente/cama, etc.). El intensivista pediátrico es consciente de que es necesario hacer cada vez más rentables las unidades de cuidados intensivos pediátricos ${ }^{4}$.

La calidad no es una moda, como se dice en la actualidad algo peyorativamente. Es también un imperativo ético, ya que no basta dar servicios, sino también satisfacer las necesidades. La calidad de atención se mide habitualmente por solo una de sus variables: calidad científico-técnica, pero esta restringida conceptualización de la calidad deja de lado variables destacadas como la accesibilidad, la oportunidad, la adecuación, la eficiencia y, sobre todo, los aspectos psicosociales de la atención al niño críticamente enfermo que deben ser tenidos en cuenta en un enfoque más holístico ${ }^{4-6}$.

El intensivista pediátrico no puede obviar entender al niño en su condición de ser concreto que pertenece a una familia y un grupo social, en resumen un ser histórico en el sentido individual y social. Es preciso potenciar, en la mayor medida posible, la humanización de la asistencia intensiva al niño para contrarrestar la frialdad inherente a la unidad de cuidados intensivos pediátricos y unida al desarrollo tecnológico. La colaboración de los padres deberá ser cada vez mayor, permitiendo su entrada libre en la UCl y su colaboración en pequeñas actividades que los vinculen, en la medida de lo posible, con el tratamiento de su hijo ${ }^{7,8}$.

Los sentimientos de la familia oscilan en gran medida entre un sentimiento de aceptación de la enfermedad y otro de rechazo. En estas circunstancias, es muy frecuente que la familia cree su propios mecanismos de defensa, agarrándose a aspectos de la información que le resultan positivos, siendo relativamente frecuente que, tras una información exhaustiva a los padres de un niño críticamente enfermo, estos, al final de la información, se queden con el aspecto más positivo de ella o aquel otro totalmente secundario e intrascendente en el grave contexto del paciente, pero que para ellos constituye un elemento que les permite mantener esperanzas. La interrelación con las familias requiere perfilar técnicas de comunicación cada vez más óptimas ${ }^{7,8}$.

La determinación de terminalidad en una UCIP es, en muchas ocasiones, una condición de extrema dificultad, ya que implica establecer con relativa seguridad que un paciente es irrecuperable. En el caso de las UCIP, esta cuestión es mucho más difícil, ya que los niños tienen una gran capacidad de recuperación $y$, muchas veces, la evolución de las enfermedades infantiles es sorprendente, tanto en el pronóstico vital como en las potenciales secuelas. Con cierta frecuencia, los tratamientos agresivos no se abandonan hasta que la muerte es inminente, por el miedo real a etiquetar a un paciente potencialmente curable como terminal, y puede prolongarse, de esta forma, el tratamiento más allá de toda esperanza razonable. Por lo tanto, es necesario seguir profundizando en el establecimiento de criterios fiables de limitación terapéutica ${ }^{9-12}$.

Pocas personas negarían que la muerte de un niño es un gran trauma para una familia. Sin embargo, es menos frecuente que la gente reconozca que la muerte de un niño también pueda suponer un trauma para los profesionales que se ocuparon en atender al niño. Todavía más significativo es el impacto que estos profesionales van acumulando por los sucesivos fallecimientos de niños. La prevención y tratamiento del bournout es indispensable para mantener la calidad asistencial y los resultados, y evitar abandono del trabajo de los actuales o futuros intensivistas pediátricos $^{13,14}$.

A pesar de que el título de especialista en cuidados intensivos pediátricos no es un objetivo anhelado en estos momentos, al no aportar especiales ventajas para los profesionales pediátricos al atarlos excesivamente a una especialidad de singular dureza y reducido campo de actuación, el trabajo de las UCIP debería estar regulado mediante titulaciones dadas por instituciones académicas y gubernamentales, tanto para garantizar unos estándares mínimos en la asistencia como para delimitar las áreas de actuación e incentivar el desarrollo y la docencia de la especialidad, aparte de proteger a los titulados ante posibles demandas asistenciales que pudieran surgir. En Estados Unidos, la especialización en cuidados intensivos pediátricos tiene la categoría de subespecialidad, reconocida por el Americam Board of Medical Specialties, disponiendo de una gran vitalidad tanto asistencial como académica. En España, la Asociación Española de Pediatría (AEP) y la Sociedad Española de Medicina Intensiva y Unidades Coronarias SEMICYUC están trabajando en la línea de que, respetando la titulación básica (pediatra), los profesionales que así lo acrediten tengan un reconocimiento gubernamental para lograr el objetivo de promover el desarrollo de la especialidad y evitar el vacío existente en este tema.

En cuanto a la enseñanza del manejo de situaciones clínicas, la seguridad del paciente constituye uno de los principales objetivos de los sistemas sanitarios y, entre otras recomendaciones para su mejora, destaca el establecimiento de programas de entrenamiento de equipos de trabajo con simulación médica. Esta se define como una situación o lugar creados para permitir que un grupo de personas experimenten una representación de un acontecimiento real con el propósito de practicar, aprender, evaluar o entender sistemas o acciones humanas. Este debe ser el modo de adquirir habilidades en situaciones graves y poco frecuentes, sin perjuicio para el paciente ${ }^{15}$.

Los estudios clínicos multicéntricos han demostrado ser una herramienta muy útil para definir eficacias terapéuticas en enfermedades de baja prevalencia. Deberán seguir siendo potenciados para avanzar en el conocimiento.

Se están definiendo de forma muy especial los factores de prevención de las infecciones nosocomiales y se promueven nuevos protocolos diagnósticos y terapéuticos, cada vez más contrastados y simplificados, sobre la base de la evidencia clínica ${ }^{16,17}$

Del logro de una asistencia intensiva mejor surge una supervivencia mayor de niños con afección crónica, subsidiarios de asistencia mecánica de funciones orgánicas, con el consiguiente bloqueo de camas. Se impone la necesidad de extender nuestra asistencia en el ámbito extrahospitalario. 
Esta realidad actual la asumimos como propia. Surgen los niños «dependientes de tecnologías» que precisan un abordaje multidisciplinario, pues contempla la asistencia biopsicosocial a estos niños y sus familias; el entrenamiento y aceptación familiar; la asistencia técnica correspondiente; el soporte nutricional y farmacológico, con una vía de conexión directa e inmediata con el hospital. Las experiencias conocidas en este terreno son ya muchas y muy favorables. La integración en el núcleo familiar de estos niños es muy satisfactoria, tanto física como psíquicamente, para ellos y sus allegados. Para el niño y sus familias se ha comprobado una mejor calidad de vida, con una menor carga social; para nuestras unidades asistenciales, una optimización de recursos, con menor carga psicológica para el personal y, finalmente, para la administración, un menor $\operatorname{coste}^{18}$.

No se puede dejar de hacer una breve alusión a nuestro «brazo ejecutor». De poco o nada sirven nuestros conocimientos, una adecuada interpretación de datos y una intervención terapéutica muy eficaz si no tenemos quien los recoja, aplique e instaure; en ello. los recursos humanos de enfermería son nuestra pieza fundamental. La especialización de este sector sanitario será pieza fundamental para el avance de los cuidados intensivos pediátricos.

Por lo tanto, en el futuro de las unidades de intensivos pediátricos, se vislumbra una optimización en la obtención de datos. Menor invasividad y lesividad y mayor efectividad. Se tratará de pensar en el niño antes que en la propia enfermedad. Se pretenderá aplicar tratamientos científicamente correctos y contrastados. Se tratará de elaborar protocolos multicéntricos que cotejen experiencias y reduzcan los márgenes de error. Será necesario procurar un entorno adecuado al niño y su familia, cambiando el medio ambiente de la propia unidad de cuidados intensivos, readaptando nuestros esquemas clásicos de rigurosa circulación codificada y abriendo nuestras unidades a padres y familiares allegados que, con su proximidad, comprendan mejor el problema de su niño en estado crítico y nuestros desvelos en su cuidado. La seguridad del paciente se garantizará con la enseñanza en simuladores y entornos de realidad virtual. En conjunto, intentaremos ser tan humanos como científicos y técnicos.

\section{Bibliografía}

1. Lopez Herce J, Sancho M, Martino JM. Informe de la Sociedad Española de cuidados intensivos pediátricos. Distribución de los cuidados intensivos en España. An Esp Pediatr. 1999;50: 14-6.

2. López Álvarez JM, Valerón Lemaur ME, Pérez Quevedo O, Limiñana Cañal JM, Jiménez Bravo de Laguna A, Consuegra Llapurt E, et al. Traumatismo craneoencefálico pediátrico grave (I). Epidemiología, clínica y evolución. Med Intensiva. 2011;35:326-31.
3. López Álvarez JM, Valerón Lemaur ME, Pérez Quevedo O, Limiñana Caña JM, Jiménez Bravo de Laguna O, Consuegra Llapurt E, et al. Traumatismo craneoencefálico pediátrico grave (II): factores relacionados con la morbilidad y mortalidad. Med Intensiva. 2011;35:332-8.

4. García S, Ruza F. Aspectos económicos en CIP. En: Ruza F, editor. Cuidados Intensivos Pediátricos. 3. ${ }^{a}$ ed. Madrid: NormaCapitel; 2003.

5. Mencía Bartolomé S, Lopez Herce Cid J, Carrillo Álvarez A, Bustinza Arriortúa A, Moral Torrero R, Sancho Pérez L, et al. Evaluación de un programa de formación en cuidados intensivos pediátricos para residentes de pediatría. An Pediatr (Barc). 2010;73:5-11.

6. Mata Zubillaga D, López de Armentía SL, Rodríguez Lage C, Álvaro Iglesias E. Adecuación de las prescripciones farmacéuticas en una unidad de cuidados intensivos neonatales. An Pediatr. 2009;71:201-8.

7. Bartel DA, Engler AJ, Natale JE, Misra V, Lewin AB, Joseph JG. Working with families of suddenly and critically ill children: physician experiences. Arch Pediatr Adolesc Med. 2000;154:1127-33.

8. Gilmer MJ. Pediatric palliative care: a family-centered model for critical care. Crit Care Nurs Clin North Am. 2002;14:207-14.

9. Casanueva Mateos L, Ruiz López P, Sánchez Díaz JI, Ramos Casado MV, Belda Hofheinz S, Llorente de la Fuente A, et al. Cuidados al final de la vida en la unidad de cuidados intensivos pediátrica. Revisión bibliográfica. An Pediatr (Barc). 2005;63:152-9.

10. Levy M. End-of-life care in the intensive care unit: Can we do better? Crit Care Med. 2001;29 Suppl:N56-61.

11. Martino Alba R, Casado Flores J, Ruiz Díaz MA. Actitudes y necesidades de los intensivistas pediátricos ante la muerte de sus pacientes. An Pediatr. 2007;66:351-6.

12. Palomeque Rico A. Cuidados intensivos y cuidados paliativos. An Pediatr (Barc). 2005;62:409-11.

13. Ullrich CK, Maye $\mathrm{OH}$. Fatiga por compasión del personal de los cuidados intensivos pediátricos. Pediatr Clin N Am. 2007;54:1005-26.

14. Bustinza Arriortua A, López-Herce Cid J, Carrilla Álvarez A, Vigil Escribano MD, De lucas García N, Panadero Carcavilla E. Situación de bournout de los intensivistas pediátricos españoles. An Pediatr. 2000;52:418-23.

15. Gonzalez JM, Chaves J, Ocete E, Calvo C. Nuevas metodologías en el entrenamiento de emergencias pediátricas: simulación médica aplicada a pediatría. An Pediatr. 2008;68:612-20.

16. Jordan García I, Bustinza Arriourtúa A, Concha Torre JA, Gil Antón J, De Carlos Vicente JC, Téllez González C. Estudio Multicéntrico nacional sobre las infeccion nosocomial en UCIP. An Pediatr. 2011, doi:10.1016/j.anpedi.2010.09.010.

17. Flores-González JC, Hernández-González A, Rodríguez-López C, Roldán-Cano V, Rubio-Quiñones F, Quintero-Otero S, et al. Infección nosocomial del tracto urinario en niños críticos. Med Intensiva. 2011;35:339-43.

18. De Oliveira Rios dos Santos MH, Redondo Collazo l, Oreiro Vilacoba MD, Rodríguez Núñez A. Ventilación domiciliaria invasiva. Perspectiva de los padres. An Pediatr. 2007;67:613-4. 